\title{
Effect of Recombination between a Molecular Ion and an Electron on Radial Dose in the Irradiation of a Heavy Ion
}

\author{
Kengo Moribayashi ${ }^{1}$ \\ ${ }^{1}$ Japan Atomic Energy Agency, 8-1-7, Umemidai, Kizugawa-city, 619-0215, Japan \\ Correspondence: Kengo Moribayashi, Japan Atomic Energy Agency, 8-1-7, Umemidai, Kizugawa-city, 619-0215, \\ Japan. E-mail: moribayashi.kengo@jaea.go.jp
}

Received: November 26, 2015

Accepted: December 28, 2015 Online Published: January 29, 2016

doi:10.5539/apr.v8n1p138

URL: http://dx.doi.org/10.5539/apr.v8n1p138

\begin{abstract}
This paper discusses the effect of recombination between a molecular ion and a free electron on radial dose in the irradiation of a heavy ion through simulations. This irradiation produces molecular ions and free electrons due to the heavy ion impact ionization. The composition electric field, which is formed from these molecular ions, traps some of free electrons and these trapped free electrons increase radial dose near the heavy ion path. These trapped electrons also cause the recombination and that the recombination enhances radial dose.
\end{abstract}

Keywords: heavy ion irradiation, radial dose simulation, recombination, cancer therapy

\section{Introduction}

Radial dose (or local dose) distributions are incorporated into the treatment planning system for heavy particle cancer therapy in order to estimate the cell survival (Kase et al., 2008). Here, the radial dose is the dose for the exposure of a heavy ion onto matter as a function of distances from the incident ion path $(r)$. The radial dose employed in this system should achieve as high accuracy as possible because it is related to the life and death of a human being. Therefore, we have proposed a radial dose simulation model (Moribayashi, 2014a, 2015a, 2015 b) that overcomes weak points of the paradigms (or widely accepted models) employed to solve the radial dose distribution in this system at present. Our model (Moribayashi, 2011, 2013a) may allow us to treat the physical phenomena due to the exposure of a heavy ion closer to reality than those produced from the paradigms. To further develop our model, we incorporate the recombination between a molecular ion and a free electron onto our model in this paper. Here, a free electron is defined as the electron ionized from a target molecule.

Two types of radial dose distributions have been available (Kase et al., 2008), where we call them 'Katz type distribution' (Katz \& Kobetich, 1969; Kraft et al., 1992) and 'Chatterjee type distribution' (Chatterjee \& Schaefer, 1976; Magee \& Chatterjee, 1980), respectively in this paper after the name of first developers of these distributions. There is a different feature in the region near the incident ion path between these distributions although this region is the most important to estimate the cell survival (Kase et al., 2008). This difference arises from the treatment of the ionization energy of a molecule and the electric field due to the polarization (Lindhard \& Winther, 1964; Ziegler et al., 2008) induced from the exposure of a heavy ion according to paradigms. The paradigms employed in these distributions are the free electron gas (Lindhard \& Winther, 1964; Ziegler et al., 2008) and the binary collision models (Katz \& Kobetich, 1969; Kraft et al., 1992), respectively. In the Chatterjee type distribution (Chatterjee \& Schaefer, 1976; Magee \& Chatterjee, 1980) where the free electron gas model is employed, the electric field due to the induced polarization produces plasma oscillation that enhances the radial dose. However, in this model, the ionization energy of a molecule in the target is ignored. In the Katz type distribution where the binary collision model is employed, the accurate ionization energies are treated, but the induced polarization is neglected. Due to this neglect, the plasma oscillation is not formed and the radial dose near the heavy ion path becomes much smaller than the Chatterjee type distribution (Kase et al., 2008). Zigler et al. (2008) wrote '(Felix) Bloch showed that the Bohr classical solution was valid for hard close collision while the Bethe solution was valid for weak scattering. Bloch then provided a solution which reduced to the Bethe solutions for hard collisions and almost reduced to the Bohr solutions for weak scattering. The terms "hard" and "weak" refer to the amount of energy transfer and are not well defined.' Here, the Bohr and the Bethe solutions mean the solutions solved from the free electron gas and the binary collision models developed around 1940s, respectively. The solution provided by Bloch indicates that we must consider the ionization energy of a molecule 
in the weak scattering because it becomes comparable with the energy transfer. It should be noted that the free electrons, which are produced from the weak scattering, forms the plasma oscillation and that this oscillation enhances radial dose in the Chatterjee type distribution (Chatterjee \& Schaefer, 1976; Magee \& Chatterjee, 1980).

In our model, which is based on the binary collision model, we consider not only the impact processes between each individual charged particle (ion, free electron etc.) and each individual molecule but also the movement of each individual free electron controlled by the Coulomb interaction of the charged particles (Moribayashi, 2011, 2013a). This interaction creates strong local electric field, which is mainly formed from molecular ions due to incident ion impact ionization, near the incident ion path and this electric field traps some free electrons. We have found that these trapped electrons enhance radial dose (Moribayashi, 2014a, 2015a, 2015b), that is, play a similar role to the plasma oscillation in the Chatterjee type distribution.

\section{Preliminary Theory}

We treat water as a target in this paper because water molecules are the largest component in a biological cell. The rough scenario of the production process of radial dose is as follows. (1) A heavy ion irradiates water and interacts with water molecules. (2) This interact produces free electrons. (3) The radial dose is obtained from the energy transfer from these free electrons to the target molecules. This energy transfer is caused from the following electron impact processes. (I) Ionization from the ground state of a water molecule $\left(\mathrm{e}^{-}+\mathrm{H}_{2} \mathrm{O} \rightarrow \mathrm{e}^{-}+\right.$ $\mathrm{H}_{2} \mathrm{O}^{+}+\mathrm{e}^{-}$) (Orient \& Srivastava, 1987), (II) electronic excitation $\left(\mathrm{e}^{-}+\mathrm{H}_{2} \mathrm{O} \rightarrow \mathrm{e}^{-}+\mathrm{H}_{2} \mathrm{O}^{*}\right.$ ) (Pritchard, et al., 1990), and (III) ionization from a excited state of a water molecule $\left(\mathrm{e}^{-}+\mathrm{H}_{2} \mathrm{O}^{*} \rightarrow \mathrm{e}^{-}+\mathrm{H}_{2} \mathrm{O}^{+}+\mathrm{e}^{-}\right)$(Moribayashi, 2011). In this paper, we also consider (IV) recombination between an ion and a free electron $\left(\mathrm{H}_{2} \mathrm{O}^{+}+\mathrm{e}^{-} \rightarrow \mathrm{H}_{2} \mathrm{O}^{*}\right)$ (Fujimoto, 2004, Landshoff \& Perez, 1976, Hahn, 1997), where $\mathrm{H}_{2} \mathrm{O}$ and $\mathrm{H}_{2} \mathrm{O}^{*}$ is the ground and excited states of water molecule, respectively.

\subsection{Recombination}

There are some types of the recombination processes in plasma (Fujimoto, 2004, Landshoff \& Perez, 1976, Hahn, 1997), that is, radiative recombination (RR) ( $\left.\mathrm{e}^{-}+\mathrm{H}_{2} \mathrm{O}^{+} \rightarrow \mathrm{H}_{2} \mathrm{O}^{*}+h v\right)$, three body recombination (TBR) ( $\mathrm{e}^{-}+\mathrm{e}^{-}$ $\left.+\mathrm{H}_{2} \mathrm{O}^{+} \rightarrow \mathrm{e}^{-}+\mathrm{H}_{2} \mathrm{O}^{*}\right)$, and dielectric recombination (DR) $\left(\mathrm{e}^{-}+\mathrm{H}_{2} \mathrm{O}^{+} \rightarrow \mathrm{H}_{2} \mathrm{O}^{* *} \rightarrow \mathrm{H}_{2} \mathrm{O}^{*}+h v\right)$, where and $\mathrm{H}_{2} \mathrm{O}^{* *}$ is the auto-ionization state of $\mathrm{H}_{2} \mathrm{O}$ and $h v$ is the photon. In the radiation chemistry, the other type of recombination exists, that is, the so-called geminate ion recombination such as $\mathrm{e}^{-}+\mathrm{H}_{2} \mathrm{O}+\mathrm{H}_{2} \mathrm{O}^{+} \rightarrow \mathrm{H}_{2} \mathrm{O}+\mathrm{H}_{2} \mathrm{O}$ (Warman, et al., 1979). However, we may be able to ignore the geminate ion recombination processes because they are considered to take place only after $T_{e}$ decreases to a room temperature $(\sim 300 \mathrm{~K})$. For the radial dose simulation, $T_{e}$ should be larger than $10,000 \mathrm{~K}$ because free electrons should have enough energy to cause impact ionization (or excitation) of a water molecule (Moribayashi, 2014a, 2014b).

The recombination processes are determined from the detailed balance principle (or theorem) (Rodberg \& Thaler, $1967)$ using cross sections or rates. Using inverse processes, the cross sections $\left(\sigma_{r}\right)$ for the recombination processes are given by (Hahn, 1997; Rodberg \& Thaler, 1967)

$$
\sigma_{r}=\frac{v_{e i} g_{e i}}{v_{e r} g_{e r}} \sigma_{i}
$$

where $v_{\text {er }(e i)}$ and $g_{e r(e i)}$ are the electron velocity and the spin degeneracy of the electronic state of a water molecule before the recombination (the inverse process), respectively, and $\sigma_{i}$ is the cross sections of the inverse process. The inverse processes for TBR and RR are the electron impact ionization and the photo-absorption, respectively. We can calculate the recombination rates (Moribayashi et al., 1998, Fujimoto, 2004, Landshoff \& Perez, 1976, Hahn, 1997) from $T_{e}, E_{e}, D_{e}, v_{e}$, and $\sigma_{r}$, where $T_{e}, E_{e}, D_{e}$, and $v_{e}$ are the temperature, the energy, the density, and the velocity of the free electrons, respectively. For the plasma produced from the irradiation of a heavy ion, we have estimated $T_{e} \leq 10 \mathrm{eV}$ and $D_{e} \leq 10^{21} / \mathrm{cm}^{3}$ near the incident heavy ion path (Moribayashi, 2014b). When $T_{e}=$ $10 \mathrm{eV}$ and $D_{e}=10^{21} / \mathrm{cm}^{3}$, we estimate the rates (or the typical reaction times) of DR, RR, and TBR to be $\sim 10^{10}$ $\mathrm{s}^{-1}(\sim 100 \mathrm{ps}), \sim 5 \times 10^{7} \mathrm{~s}^{-1}(\sim 20 \mathrm{ps})$, and $\sim 5 \times 10^{13} \mathrm{~s}^{-1}(\sim 20 \mathrm{fs})$, respectively. These estimations are obtained from Figure 13 shown by Hahn (1997) (where DR of $\mathrm{F}^{+}$ion is treated) and Figure 4B.7 shown by Fujimoto (2004). We treat only TBR in this paper because radial dose has been determined from the physical processes, that is, we treated up to $t \sim 100 \mathrm{fs}$ (Moribayashi, 2014a, 2015a), where $t$ is the time after the irradiation of a heavy ion. Fujimoto (2004) has showed that TBR should produce only excited states of a molecule.

\subsection{Rate Equations}

We study recombination processes using a simulation model that treats individual free electrons and individual molecules for the first time as far as we know. This allows us to treat recombination in the local small size 
regions where $D_{e}$ changes according to $r$. This model may be suitable for radial dose simulations, but the recombination processes have been treated only by rate equations before this paper. Firstly, we have treated the rate equations (Moribayashi et al., 1998, 1999; Moribayashi, 2007) to elucidate the role of the recombination processes. Suppose that $i$ expresses the state of a water molecule and that $i=1,2$, and 3 correspond to the states of $\mathrm{H}_{2} \mathrm{O}, \mathrm{H}_{2} \mathrm{O}^{*}$, and $\mathrm{H}_{2} \mathrm{O}^{+}$, respectively. Then, the rate equations for processes (I - IV) are written by

$$
\begin{aligned}
& \frac{d P_{1}}{d t}=-\left(\alpha_{12}+\alpha_{13}\right) P_{1}, \\
& \frac{d P_{2}}{d t}=\alpha_{12} P_{1}-\alpha_{23} P_{2}+\alpha_{32} P_{3}, \\
& \frac{d P_{3}}{d t}=\alpha_{13} P_{1}+\alpha_{23} P_{2}-\alpha_{32} P_{3},
\end{aligned}
$$

where $\alpha_{i j}$, and $P_{i}$ are the transition rate from the $i^{\prime}$ th to the $j^{\prime}$ th states and the population of the $i^{\prime}$ th state, respectively. Using Maxwell distribution function for $T_{e}$ and $E_{e}$ (Moribayashi, 2014a, 2014b)

$$
f\left(T_{e}, E_{e}\right)=A \sqrt{E_{e}} \exp \left(-\frac{E_{e}}{T_{e}}\right)
$$

the rates of electron impact ionization and excitation processes $\left(\alpha_{12}, \alpha_{13}, \alpha_{23}\right)$ are given by (Moribayashi et al., 1998, 1999, Moribayashi, 2007)

$$
\alpha_{i, j}=D_{e} \int_{0}^{\infty} f\left(T_{e}, E_{e}\right) \sigma_{i j}\left(E_{e}\right) v_{e} d E_{e},
$$

where $\sigma_{i j}$ is the transition cross section from the $i$ 'th to the $j$ 'th states, respectively. From equation (2), the change of $T_{e}$ between times of $t$ and $t+\Delta t$ can be written by (Moribayashi, 2014b)

$$
T_{e}(t+\Delta t) \sim \frac{2\left[\frac{3}{2} T_{e}(t) D_{e}(t)-\Delta t\left\{V_{I} \alpha_{13} P_{1}+V_{I I} \alpha_{12} P_{1}+V_{I I I} \alpha_{23} P_{2}+V_{I V} \alpha_{32} P_{3}\right\}\right.}{3 D_{e}(t+\Delta t)},
$$

where $V_{i}$ is the transition energy due to process $(i)$. For processes (I) - (IV), we take $V_{i}$ to be 12.6, 10.24, 2.36, and $-2.36 \mathrm{eV}$, respectively. It should be noted that $V_{\mathrm{IV}}$ becomes a negative value because TBR reduces the internal energies of a water molecule. Here, the first term of the numerator of the right side of this equation is obtained from the averaged electron energy $\left(E_{\text {ea }}\right)$ per unit area given by (Moribayashi, 2014b)

$$
E_{e a}=\frac{\int_{0}^{\infty} E_{e} f\left(E_{e}\right) d E_{e}}{\int_{0}^{\infty} f\left(E_{e}\right) d E_{e}}=\frac{3}{2} T_{e} .
$$

\section{Simulation Model}

We have employed our Monte Carlo (MC) model (Moribayashi, 2011, 2013a) for radial dose simulations (Moribayashi, 2014a, 2015a, 2015b). A lot of Monte Carlo simulations, which are based on the binary collision model, have been executed. As far as we know, the secondary electrons only had gone away from the incident heavy ion path (Katz \& Kobetich, 1969, Kraft et al., 1992, Nikjoo et al., 1998, Uehara \& Nikjoo, 2002, Wang \& Vassiliev, 2014) because the polarization induced from the heavy ion irradiation had not been considered. In our model, we treat individual molecules, the movements of individual free electrons (see section 3.2) and the induced polarization (see section 3.3). This polarization forces some free electrons to return to the region near the heavy ion path. We also show the calculation method of the radial dose (see section 3.4) and the verification of our model (see section 3.5). More details are shown in our previous papers (Moribayashi, 2011, 2013a).

\subsection{Procedures of Our Simulation Model}

The procedures of our simulation model are shown as follows: procedure (i) we set up positions of water molecules to obtain the density of liquid water in the target. The time $(t)$ and the number of free electrons $\left(N_{e}\right)$ are set to 0 . (ii) A heavy ion moves in the target. (iii) We examine the change of states of water molecules according to this heavy ion impact. (iv) When we judge that ionization occurs, we produce a molecular ion and a 
free electron and add 1 to $N_{e}\left(N_{e}=N_{e}+1\right)$. (v) We also determine an initial energy and an emission angle of this free electron from the experimental data. (vi) When $N_{e}>0$, we examine the change of states of water molecules according to free electron impact processes. (vii) Procedures (iv) and (v) are executed when we judge that ionization occurs. When we judge that recombination takes place, we set the charge of the molecule of interest to 0 and $N_{e}=N_{e}-1$. (viii) Suppose that $i$ is the process number that occurs. Then, we change the energy of the free electrons according to process $(i)$ and record $i, t, r$, and $V_{i}$. These records are employed for the simulation of the radial dose distribution. (ix) The electric field due to the polarization induced in the target is calculated. The changes of the free electron velocities due to this electric field are simulated using Newton's equations (Jurek et al., 2004, Moribayashi, 2009, 2010). (x) We add $\Delta t$ to $t(t=t+\Delta t)$ and move an incident ion and free electrons according to their velocities. (xi) Procedures (iii) - (x) are executed. (xii) When $t$ becomes $t_{\max }$, the other heavy ion irradiates onto a different place and procedures (i) - (xi) are repeatedly executed for one hundred times. We show the results averaged over the radial dose produced from the irradiation of these one hundred ions for every $E_{\text {ion }}$ values in section 4 , where $E_{\text {ion }}$ is the energy of this ion.

\subsection{The Explanation of Procedures (ii - v)}

We employ total impact ionization cross sections of an incident heavy ion for the production of molecular ions and free electrons (Cappello et al., 2009). The centers of these cross sections are located at the center of the molecules and these cross sections are perpendicular to the direction of the velocity of the incident heavy ion. It is judged that ionization occurs only when this heavy ion crosses the area of these cross sections (Jurek et al., 2004; Moribayashi, 2009, 2010, 2011).

From the measurement values of differential ion impact ionization cross sections (Champion et al., 2007, Cappello et al., 2009, Ohsawa et al., 2013), we determine an initial energy $\left(E_{e}\right)$, an emission angle of elevation $(\theta)$, and an azimuth $(\phi)$ of the ionized electrons by solving an equation for a variable $x\left(=E_{e}\right.$, or $\theta$, or $\left.\phi\right)$, that is,

$$
\frac{\int_{0}^{x} f\left(x^{\prime}\right) d x^{\prime}}{\int_{0}^{x_{\max }} f\left(x^{\prime}\right) d x^{\prime}}=R_{n},
$$

where $f\left(x^{\prime}\right), x_{\max }$, and $R_{n}$ are the distribution function, the maximum value of $x$, and the random number, respectively. Using $R_{n}, x$ varies according to events of ion impact ionization. For the distribution for $E_{e}$, we employ the Rudd model (Rudd et al., 1992) that almost reproduces this distribution in $\mathrm{C}^{6+}$ ion impact ionization (Cappello et al., 2009, Ohsawa et al., 2013). We take the distribution for $\theta$ and $\phi$ to be isotropic (Moribayashi, 2014a, 2015a). For $\theta$ and $\phi, f(\theta)=\sin \theta$ and $f(\phi)=1$ because we employ the differential cross sections per a solid angle (Champion et al., 2007, Cappello et al., 2009).

\subsection{The Explanation of Procedure (ix)}

For the movement of the free electrons, we consider the following force acting on the free electrons;

$$
\vec{F}=m_{e} \frac{d \vec{v}_{e i}}{d t}=-\sum_{j \neq i} \frac{e^{2} \vec{r}_{i j}}{4 \pi \varepsilon_{0} r_{i j}^{3}}+\sum_{l} \frac{q_{l} e \vec{r}_{i l}}{4 \pi \varepsilon_{0} r_{i l}^{3}},
$$

where $\varepsilon_{0}, m_{e}, \vec{v}_{e i}, q_{l}$, and $\vec{r}_{i j(l)}$ are the dielectric constant in vacuum, the mass of an electron, the velocity of the $i$ 'th electron, the charge of the $l^{\prime}$ th ion, and the distances between the $i$ 'th electron and the $j^{\prime}$ th electron (the $l^{\prime}$ th ion), respectively (Moribayashi, 2009, 2010, 2011). This force corresponds to the electric field due to the polarization, which is induced from the irradiation of a heavy ion, acting on the free electrons.

\subsection{The Explanation of Procedures (vi - viii)}

For the radial dose simulations, we employ electron impact cross sections for processes (I) - (III) shown in section 2. For these processes, we treat the same method as that of heavy ion impact ionization (see Sec.2.2) using the total cross sections of the electron impact (I) to (III). When we judge that the electron impact process occurs, we determine which process occurs using the cross sections treated with a statistical weight and $R_{n}$ from the Monte Carlo method. We record $k, r, t$, and $\Delta E_{k}$, where $k$ expresses the electron impact process and $k=\mathrm{I}$ - III (see section 2). We simulate $N_{\text {ett }}^{(k)}(r-\Delta r, r+\Delta r, t)$, where $N_{e t t}^{(k)}(r-\Delta r, r+\Delta r, t)$ is the number of times that process $k$ occurs in the region between $r-\Delta r$ and $r+\Delta r$ at $t, \Delta r$ is the interval length of $r$, and $\Delta E_{k}$ is the energy transfer to the target due to process $k$. Then, the radial dose $\left(R_{D}\right)$ is given by (Moribayashi, 2014a, 2015a, 2015b) 


$$
R_{D}(r)=\frac{\sum_{k=I}^{I I I} \Delta E_{k} N_{e t}^{(k)}(r)}{\pi\left\{(r+\Delta r)^{2}-(r-\Delta r)^{2}\right\} l d_{t a}},
$$

with

$$
N_{e t}^{(k)}(r)=\int_{0}^{t_{\text {max }}} N_{e t t}^{(k)}(r-\Delta r, r+\Delta r, t) d t,
$$

where $d_{t a}$ and $l$ are the target density and the incident heavy ion migration length, respectively.

We incorporate cross sections of TBR obtained from equation (1) into our simulation model. When the following conditions are satisfied, we assume that TBR occurs. (A) Two free electrons exist in one water molecular ion at the same time (The distances between the free electron and the center of the water molecular ion of interest are shorter than $0.15 \mathrm{~nm}$ because the mean intermolecular distance in water is about $0.3 \mathrm{~nm}$.). (B) The energy $\left(E_{1}\right)$ of the free electron, which becomes a bound electron in the water molecule due to TBR, is smaller than $V_{\text {III. }}$ We employ only the lowest electronic excited state of a water molecule in this paper. The average initial energy of the free electrons due to electron impact ionization is smaller than or almost the same as $V_{\text {III }}$ when we consider the inverse process, that is, the electron impact ionization. (III) The other electron $\left[\mathrm{e}^{-}\left(E_{3}\right)\right]$ is located within the cross sections given by equation (1), where the center of the cross section is located at the place of the center in the water molecule of interest (Moribayashi, 2009, 2010, 2011).

\subsection{Verification of our Simulations}

Here, we show the verifications that the procedures shown in section 3.1 operate well. For procedures (iii, iv, vi), we confirmed that we reproduce the mean path between ionization events $(\tau)$ due to ion (or electron) impact processes given by $\tau=(n \sigma)^{-1}$, where $n$ and $\sigma$ are the number density of molecules and the impact cross section, respectively (Moribayashi, 2011). For procedures (v, vii), we confirm the verification that equation (7) operates well in our previous paper (Moribayashi, 2015b). For procedure (viii), our radial dose simulation results (Moribayashi, 2014a, 2015a, 2015b) agree well with the conventional distributions in the region far from the ion path where the effect of the polarization disappears and the recombination seldom takes place. In this region, these conventional distributions were shown to agree well with the experimental data (Varma et al., 1977). Therefore, the agreement of our results with the conventional distributions implies that our simulation results in this region are supported by the experiment.
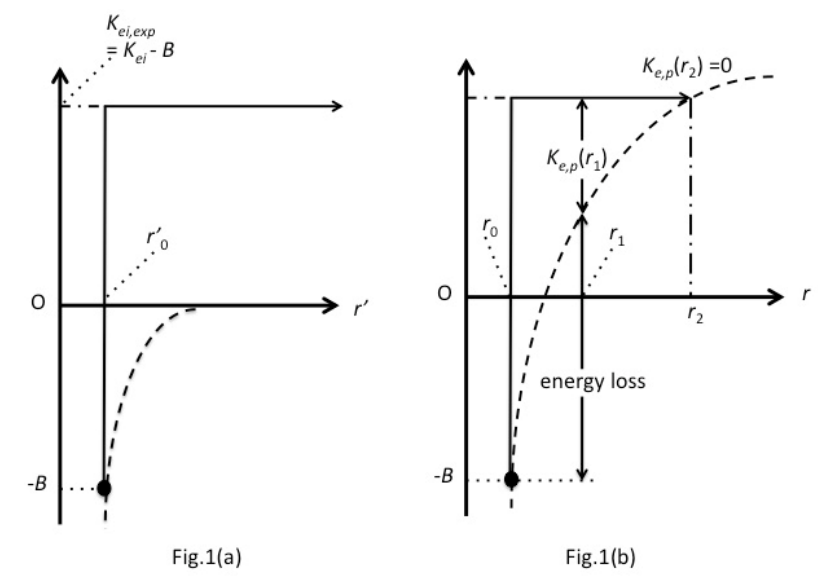

Figure 1. (a) $K_{e}$ (shown by arrows), $K_{e i}, V$ (shown by broken lines) vs. $r$ ' and (b) $K_{e, p}$ (shown by arrows), $K_{e i, p}, V_{p}$ (shown by broken lines) vs. $r$. In Figure 1(b), the position where the free electron is trapped by the potential and the relationship between the energy loss of a free electron and $V$ are shown

For procedure (ix), we performed an operation check of our simulation code for the movement of the free electrons from the change of a free electron energy shown in Figure 1 (a). Figure 1 (a) shows $K_{e}, K_{e i}$, and $V$ as a function of $r^{\prime}$, where $r^{\prime}, K_{e}, K_{e i}$, and $V$ are the distance from a single atom (or molecule), the kinetic energies of a 
free electron, the initial value of $K_{e}$ and potential energies, respectively (Moribayashi, 2013b). We have confirmed for a free electron emitted from a single hydrogen atom that the convergence value of the energy loss becomes the same as $B \sim 13.6 \mathrm{eV}$ (Moribayashi, 2013b). This energy loss is obtained from the simulation results for the change of $v_{e}$ solved by Newton's equations [equation (8)]. For the calculation of the energy loss due to the composite electric field treated here, we calculate the force between the free electron of interest and each individual charged particle using the same code as that for a single atom. Since Newton's equations [equation (8)] due to this total force allow us to calculate $v_{e}$, we have judged that our simulation code for $v_{e}$ operates well. We also checked procedure (ix) by treating the photo-electron spectra where we studied the irradiation of strong intensity x-rays such as XFEL sources onto a cluster (Moribayashi, 2009). We showed good agreement well among the spectra calculated using our MC model employed here, the rate equations [equation (2)], and an analytical solution (Moribayashi, 2009). As for the experiment related to these electron spectra, Bosted et al. (2008) measured the photo-electron spectra emitted from Ar clusters due to the irradiation of EUV (extended ultra violet) lasers. They confirmed that the energies (or the movements) of photo-electrons are affected from the charges due to the polarization induced from the irradiation of the EUV lasers. This indicates that this experiment supports our model where the movements of the free electrons are controlled by the polarization induced from the irradiation of a heavy ion [procedure (ix)].

\section{Results and Discussions}

\subsection{Electron Temperature}

Here, using rate equations and our MC model, we discuss the relationship of TBR with $T_{e}$. The free electron energies must be larger than $V_{i}$ in order to cause process $(i)$ and the cross sections of process $(i)$ become larger with increasing electron energies up to a few $10 \mathrm{eV}$. This means that radial dose increases with increasing $E_{e a}$ [see equation (6)], that is, $T_{e}$. Considering from the energy conservation law and equation (5), we expect that TBR enhances $T_{e}$ because TBR reduces the internal energies of the molecule of interest as well as $D_{e}$.

We simulate $T_{e}$ as functions of $D_{e}$ and $P_{3}$ because $D_{e}$ and $P_{3}$ increase with decreasing $E_{\text {ion }}$ (Moribayashi, 2014b). Figures 2 (a) and (b) show $T_{e}$ calculated by equations (2) including and excluding TBR for various values of $P_{3}$ as a function of $t$, respectively. Here, we set initial values of $P_{1}+P_{3}, D_{e}$, and $T_{e}$ to $3 \times 10^{22} / \mathrm{cm}^{3}, 10^{21} / \mathrm{cm}^{3}$, and 5 $\mathrm{eV}$, respectively. When TBR is ignored [Figure 2 (a)], $T_{e}$ only decreases regardless of $P_{3}$ and is much lower than that given by including TBR [Figure 2 (b)]. It is found that TBR enhances $T_{e}$ as we expect. When TBR is considered [Figure 2 (b)], $T_{e}$ depends strongly on $P_{3}$. In Figures 2 (c) and (d), we set the same conditions except for the initial value of $D_{e}$, which is taken to be $10^{20} / \mathrm{cm}^{3}$, as those in Figures 2 (a) and (b), respectively. We have found that $D_{e}$ affects little on the trends of the change of $T_{e}$.

Figure 3 shows $T_{e}$ simulated using our MC model as a function of $t$ at $E_{\text {ion }}$ of 3, 5, 10, and $15 \mathrm{MeV} / \mathrm{u}$. Until $t=30$ fs, TBR seems to affect little on $T_{e}$. However, after $30 \mathrm{fs}, T_{e}$ becomes difference between the cases including and excluding TBR for relatively small values of $E_{\text {ion }}(3 \mathrm{MeV} / \mathrm{u}$ and $5 \mathrm{MeV} / \mathrm{u})$ and this difference increases as time passes. Namely, TBR plays a role of increasing $T_{e}$, which agrees well with Figure 2. On the other hand, this difference becomes smaller with increasing $E_{\text {ion }}$ (see $10 \mathrm{MeV} / \mathrm{u}$ and $15 \mathrm{MeV} / \mathrm{u}$ ). This may be caused from the fact that both of $P_{3}$ and $D_{e}$, which are determined from the mean path between ionization events due to incident heavy ion impact, decrease with increasing $E_{\text {ion }}$.

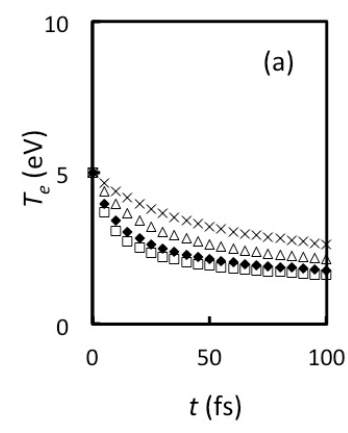

Fig.2 (a)

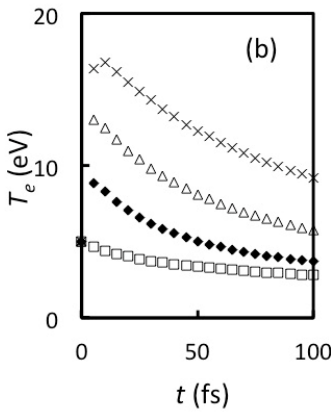

Fig.2 (b)

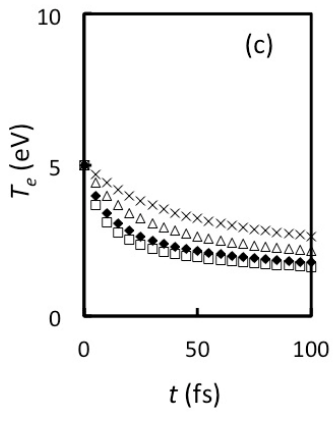

Fig.2(c)

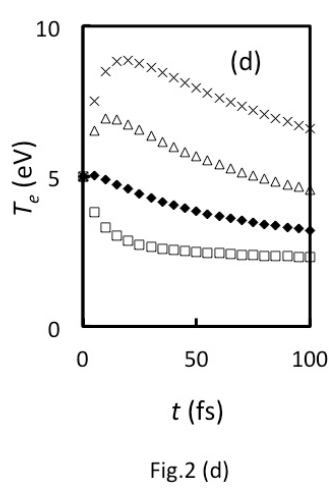

Fig.2 (d)

Figure 2. $T_{e}$ calculated from rate equations [equation (2)] vs. $t$ :the initial values of $P_{1}+P_{3}, D_{e}$, and $T_{e}$ are 3 $\times 10^{22} / \mathrm{cm}^{3}, 10^{21} / \mathrm{cm}^{3}$, and $5 \mathrm{eV}$, respectively: $D_{e i}$ is (a) $10^{21} / \mathrm{cm}^{3}$, (b) $10^{21} / \mathrm{cm}^{3}$, (c) $10^{20} / \mathrm{cm}^{3}$, and (d) $10^{20} / \mathrm{cm}^{3}$. TBR is

(a) excluded, (b) included, (c) excluded, and (d) included. $P_{3}$ is taken to be $10^{21} / \mathrm{cm}^{3}(\square), 10^{22} / \mathrm{cm}^{3}(\diamond), 2$ $\times 10^{22} / \mathrm{cm}^{3}(\triangle)$, and $2.5 \times 10^{22} / \mathrm{cm}^{3}(\times)$ 


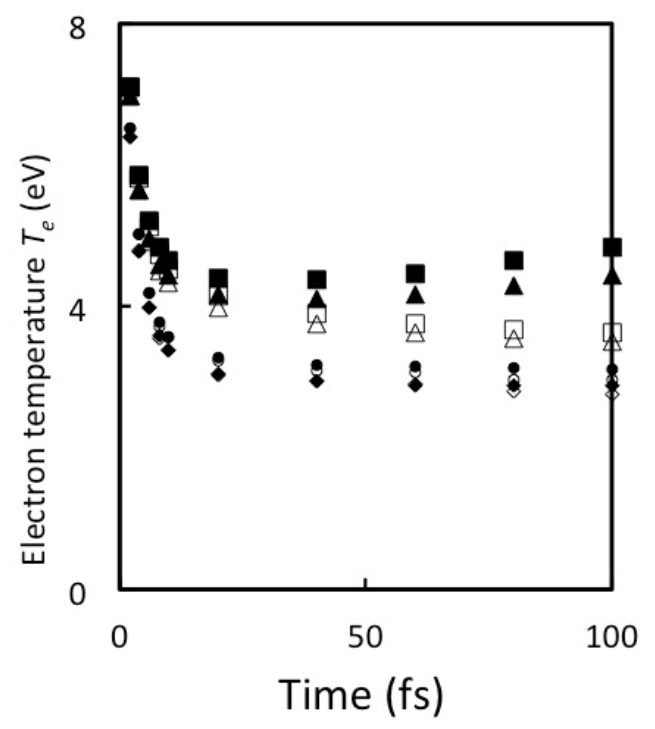

Figure 3. $T_{e}$ simulated using our MC model as a function of $t: E_{\text {ion }}$ of $3(\boldsymbol{\square}, \square), 5(\boldsymbol{\Delta}, \triangle), 10(\boldsymbol{\bullet}, \bigcirc)$, and $15 \mathrm{MeV} / \mathrm{u}$ $(\diamond, \diamond)$ : TBR is excluded $(\square, \triangle, \bigcirc, \diamond)$ (Moribayashi, 2014a) and included $(\boldsymbol{\square}, \mathbf{\Lambda}, \boldsymbol{\bullet}, \diamond)$

Although we try to treat TBR as close to reality as possible, ambiguity remains in our model such as the treatments of excited states. We have estimated the rates of TBR roughly from the number of free electrons simulated in the irradiation of a $\mathrm{C}^{6+}$ ion with the energy of $3 \mathrm{MeV} / \mathrm{u}$ from our Monte Carlo model as a function of $t$. The number of secondary electrons within the region of $r \leq 1 \mathrm{~nm}$ at $t=100 \mathrm{fs}$ becomes one third as small as the initial number, where the secondary electrons are the free electrons produced from the incident heavy ion impact ionization. This means that the timescale of the recombination is $\sim 100 \mathrm{fs}$, a little bit longer than that estimated in section $2.1(\sim 20 \mathrm{fs})$. Here, a timescale is the time when the number of the free electrons due to this process becomes $1 / \mathrm{e}$, where e is the base of natural logarithm. Such a small difference between these timescales is unavoidable and our model does not seem to overestimate the effect of TBR on radial dose. Therefore, we judge that our model can be used for our first simulation. We believe that the results and discussions shown in section 4.2 allow readers enough to understand the effect of the recombination.

\subsection{Radial Dose}

Figures 4 (a) - (f) show $R_{D}$ [equation (9)] as a function of $r$ for $E_{\text {ion }}=3,5,8,10,12$, and $15 \mathrm{MeV} / \mathrm{u}$, respectively. We take $t_{\max }$ to be $5 \mathrm{fs}, 50 \mathrm{fs}$, and $100 \mathrm{fs}$ for Figures 4 (a) and (b) and to be only $100 \mathrm{fs}$ for Figures 4 (c $-\mathrm{f}$ ). We also show our previous results (Moribayashi, 2014a) that correspond to $R_{D}$ obtained from excluding TBR and the conventional distributions (Katz \& Kobetich, 1969, Chatterjee \& Schaefer, 1976, Magee \& Chatterjee, 1980).

We have found that $R_{D}$ obtained from including TBR shows larger than that obtained from excluding TBR at $E_{i o n}$ $=3 \mathrm{MeV} / \mathrm{u}$ and $5 \mathrm{MeV} / \mathrm{u}$, that is, TBR enhances $R_{D}$. In particular, at $3 \mathrm{MeV} / \mathrm{u}, R_{D}$ obtained from including TBR becomes about 1.5 times as high as that obtained from excluding TBR. However, the effect of TBR seems to be negligible on $R_{D}$ at $E_{\text {ion }}=10 \mathrm{MeV} / \mathrm{u}$ and $15 \mathrm{MeV} / \mathrm{u}$. This may come form the fact that there is little difference between $T_{e}$ obtained from including and excluding TBR (see Figure 3).

From Figure 3 and Figure 4, we conclude that (i) the effect of TBR appears when $E_{\text {ion }}$ is relatively small, that (ii) TBR increases $T_{e}$, and that (iii) this increase enhances $R_{D}$ near the incident heavy ion path. Before our simulations, our coworkers as well as we guessed that recombination processes play a role of reducing the radial dose because the number of the free electrons decreases. However, to our surprise, we have found that recombination plays a reverse role, that is, a role of enhancing radial dose. 


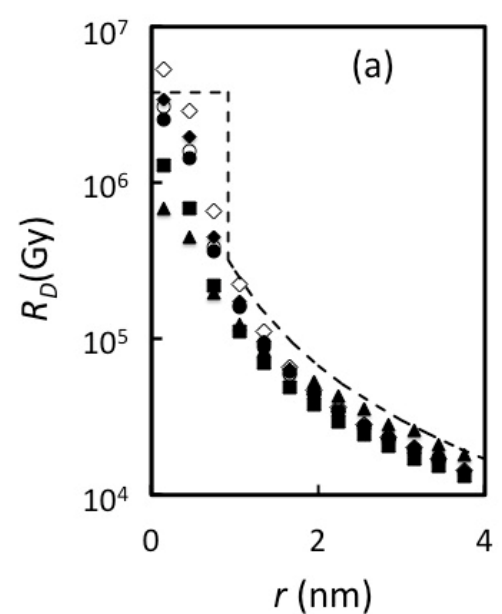

Fig.4 (a)

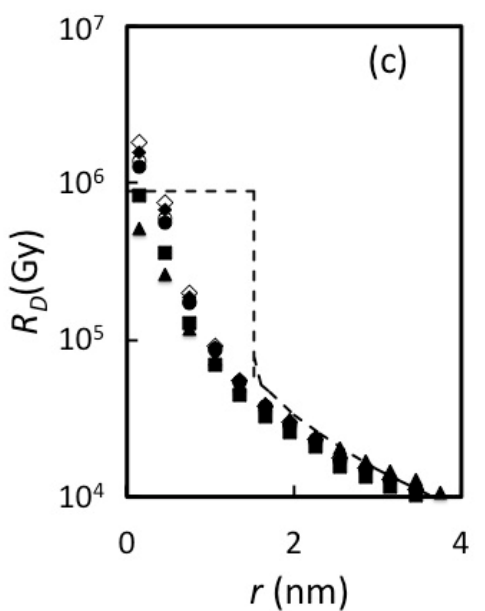

Fig.4 (c)

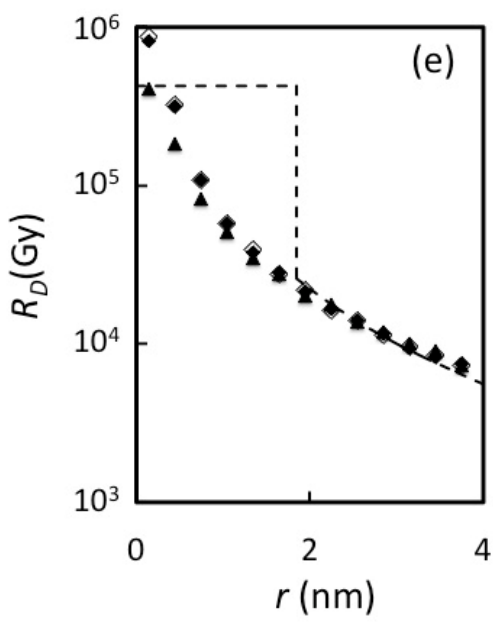

Fig.4 (e)

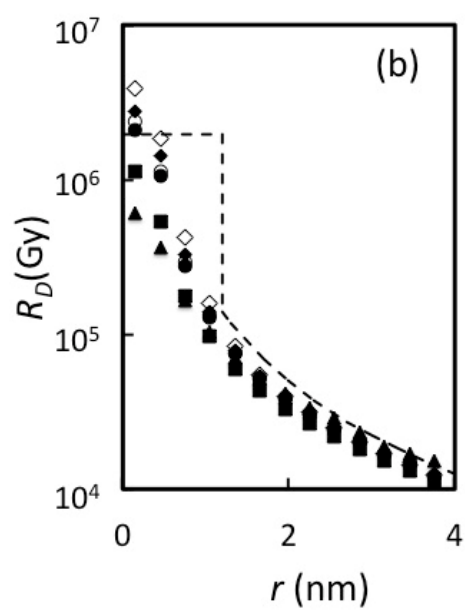

Fig.4 (b)

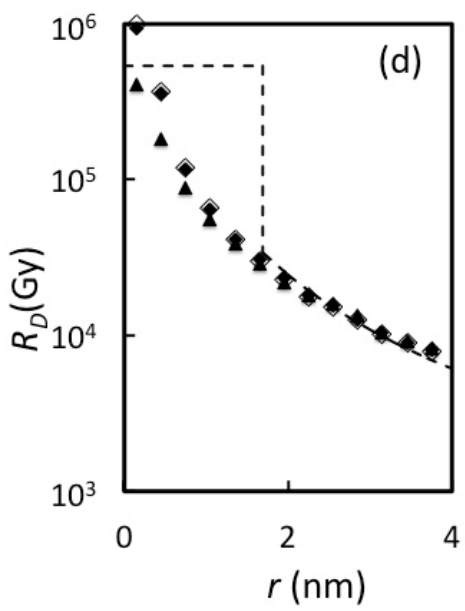

Fig.4 (d)

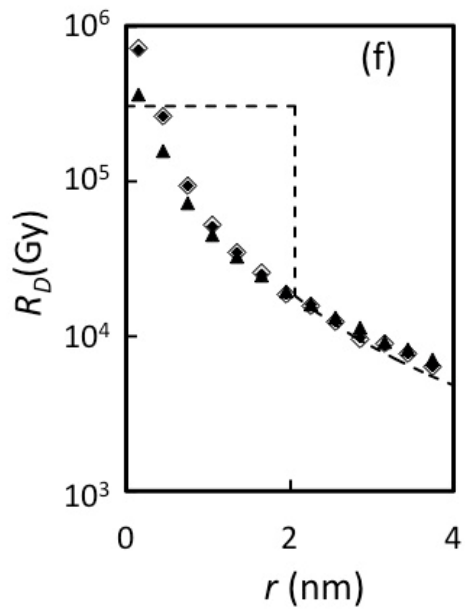

Fig.4 (f)

Figure 4. $R_{D}$ defined in equation (9) vs. $r$ for the irradiation of a $\mathrm{C}^{6+}$ ion with the energy of (a) 3 , (b) 5 , (c) 8 , (d) 10 , (e) 12 , and (f) $15 \mathrm{MeV} / \mathrm{u}$; $\mathbf{\square}: t_{\max }=5 \mathrm{fs}, \bigcirc$ and $\bigcirc: t_{\max }=50 \mathrm{fs}, \diamond$ and $\diamond: t_{\max }=100 \mathrm{fs}$. $R_{D}$ obtained from excluding $(\boldsymbol{\square}, \mathbf{\Lambda}, \mathbf{O})$ (Moribayashi, 2014a) and including $(\bigcirc, \diamond)$ three body recombination $(\mathrm{TBR})$ and the Katz type $(\mathbf{\Delta})$ (Katz \& Kobetich, 1969, Kraft et al., 1992) and Chatterjee type (--) (Chatterjee \& Schaefer, 1976, Magee \&

Chatterjee, 1980) distributions are shown 
Figure 5 shows $R_{D}$ obtained from our model and the conventional distributions at $r=0 \sim 0.3,0.3 \sim 0.6,0.6 \sim 0.9$, $0.9 \sim 1.2 \mathrm{~nm}$ as a function of $E_{i o n}$. We show our previous results (Moribayashi, 2015a) that correspond to $R_{D}$ obtained from excluding TBR and the conventional distributions (Katz \& Kobetich, 1969, Chatterjee \& Schaefer, 1976, Magee \& Chatterjee, 1980) in these figures. At $r>0.6 \mathrm{~nm}$ [see Figures 5 (c) and (d)], our results agree well with the Katz type distribution and show much smaller than the Chatterjee type distribution. In our model and the free electron gas model, $D_{e}$ decreases and remains constant values with increasing $r$, respectively. Since $R_{D}$ increases with increasing $D_{e}$, the Chatterjee type distribution where the free electron gas model is employed may be overestimated at $r>0.6 \mathrm{~nm}$. At $r<0.6 \mathrm{~nm}$ [see Figures 5 (a) and (b)], our results agree well and disagree with Chatterjee type distribution (in particular, for $E_{\text {ion }} \leq 5 \mathrm{MeV} / \mathrm{u}$ ) and Katz type distribution, respectively. This may come from the following fact. A lot of molecules are ionized due to free electron impact ionization in these regions, that is, $D_{e}$ obtained from our model may become comparable with that in Chatterjee type distribution.

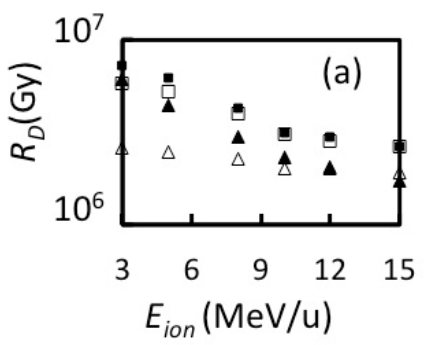

Fig.5(a)

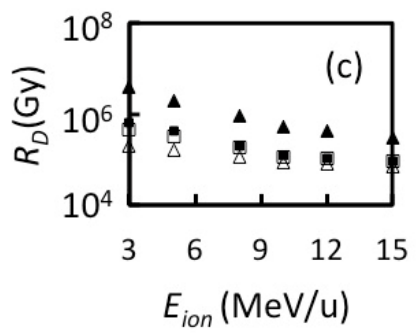

Fig.5(c)

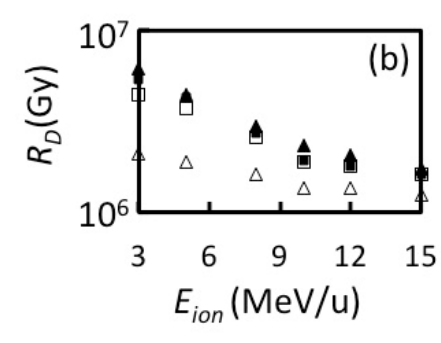

Fig.5(b)

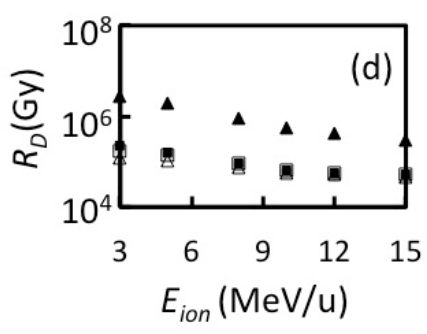

Fig.5(d)

Figure 5. $R_{D}$ defined in equation (9) at $r=0 \sim 0.3,0.3 \sim 0.6,0.6 \sim 0.9,0.9 \sim 1.2 \mathrm{~nm}$ vs. $E_{\text {ion }}$; Results obtained from including ( $\square$ ) and excluding $(\square)$ (Moribayashi, 2015a) TBR and the Katz type $(\triangle)$ (Katz \& Kobetich, 1969, Kraft et al., 1992) and Chatterjee type (A) (Chatterjee \& Schaefer, 1976, Magee \& Chatterjee, 1980) distributions are shown

\section{Summary}

We have developed a radial dose simulation model due to the exposure of a heavy ion as close to reality as possible. We incorporate three body recombination (TBR) processes where a molecular ion and two free electrons are involved into our model and study the effect of TBR on radial dose. We have found that the effect of TBR appears when the incident heavy ion energy is relatively small and that TBR enhances radial dose.

We compare radial dose obtained from our model with those from the conventional model and we suggest which conventional distributions should be selected according to $r$ and $E_{\text {ion }}$, where $r$ and $E_{\text {ion }}$ are the distance

Our simulation model, which has become possible in the $21^{\text {st }}$ century, is the only way to examine the physical phenomena that occurs near the ion path at present as far as we know. We believe that our model developed in this paper is useful for the development of atomic and molecular physics, bio-medical physics, and computational physics and become a bridge among these fields.

\section{Acknowledges}

We wish to thank Dr. D. Ohsawa (Kyoto Univ.), Dr. K. Akamatsu, Dr. J. Koga, Dr. N. Shikazono, Dr. A. Tanaka, and Director H. Namba (JAEA) for their explaining us a lot of expert knowledge of radiation science and their useful discussions. We also wish to thank Dr. Y. Iriki, Prof. H. Tsuchida, Prof. A. Ito (Kyoto Univ.) for their 
useful discussions. Our simulation in this paper has been performed using super computer 'PRIMERGY BX900' at Tokai Research and Development Center in JAEA. This work has been supported by JSPS KAKENHI Grant Number 25390131. Our simulation in this paper has been performed using super computer 'PRIMERGY BX900' at Tokai Research and Development Center in JAEA.

\section{References}

Bostedt, C., Thomas, V., \& Hoener, M. (2008). Multistep Ionization of Argon Clusters in Intense Femtosecond Extreme Ultraviolet Pulses. Phys. Rev. Lett., 100, 133401/1-4.

Cappello, C. D., Champion, C., Boudrioua, O., Lekadir, H., Sato, Y., \& Ohsawa, D. (2009). Theoretical and experimental investigations of electron emission in $\mathrm{C}^{6+}+\mathrm{H}_{2} \mathrm{O}$ collisions. Nucl. Instru. and Meth. Phys. Res. B. 267, 781-90.

Champion, C., Boudrioua, O., Cappello, D. C., Sato, Y., \& Ohsawa, D. (2007). Theoretical and experimental investigations of electron emission in $\mathrm{He}^{2+}+\mathrm{H}_{2} \mathrm{O}$ collisions. Phys. Rev. A, 75, 032724/1-9.

Chatterjee, A., \& Schaefer, H. J. (1976). Microdosimetric structure of heavy ion track in tissue. Radiat. Environ. Biophys., 13, 215 - 227.

Fujimoto, T. (2004). Plasma Spectroscopy (In J. Birman (Ed.), chap. 5). Clarendon press Oxford (Oxford science publications).

Hahn Y. (1997). Electron-ion recombination processes-an overview. Rep. Prog. Phys., 60, 691-759.

Jurek, Z., Faigel, G., \& Tegze, M., (2004). Dynamics in a cluster under the influence of intense femtosecond hard X-ray pulses. Eur. Phys. J. D., 29, 217-229.

Kase, Y., Kanai, T., Matsufuji, N., Furusawa, Y., Elsässer, T., \& Scholz, M. (2008). Biophysical calculation of cell survival probabilities using amorphous track structure models for heavy-ion irradiation Phys. Med. Biol., 53, 37 - 59.

Katz, R., \& Kobetich, E. J. (1969). Particle Tracks in Emulsion. Phys. Rev., 186, 344 -351.

Kraft, G., Krämer, M., \& Scholz, M., (1992). LET, track structure and models. Radiat. Environ. Biophys,. 31, 161-180.

Landshoff, R. K., \& J. D. Perez, (1976). Determination of plasma parameters of laser-produced aluminium plasma from x-ray line radiation. Phys. Rev. A, 13, $1619-1632$.

Lindhard, J., \& Winther, A. (1964). Stopping power of electron gas and equipartition rule. Mat. Fys. Medd. Vid. Selsk., 34, 1-21.

Magee, J. L., \& Chatterjee, A. (1980). Radiation chemistry of heavy-particle tracks 1 General considerations. $J$. Phys. Chem., 84, 3529-353.

Moribayashi, K. Sasaki, A., \& Tajima, T. (1998). Ultrafast X-Ray processes wit hollow atoms. Phys. Rev. A., 58, 2007-2015.

Moribayashi, K., Sasaki, A., \& Tajima, T. (1999). X-ray emission by ultrafast inner-shell ionization from vapors of Na, Mg, and Al. Phys. Rev. A, 59, 2732-2737.

Moribayashi, K., (2007). Multiply inner-shell excited states produced through multiple x-ray absorption relevant to x-ray pulses. Phys. Rev. A., 76, 042705/1-6.

Moribayashi, K., (2009). Application of photoelectron spectroscopy to the measurement of the flux of x-ray free-electron lasers irradiating clusters or biomolecules. Phys. Rev. A., 80, 025403/1-4.

Moribayashi, K. (2010). Spherically symmetric models for x-ray damage and the movement of electrons produced in non-spherically symmetric targets such as bio-molecules. J. Phys. B., 43, 165602/1-7.

Moribayashi, K. (2011). Incorporation of the effect of the composite electric fields of molecular ions as a simulation tool for biological damage due to heavy-ion irradiation. Phys. Rev. A., 84, 012702/1-7.

Moribayashi, K. (2013a). Movement of secondary electrons due to the irradiation of heavy ions: Role of the composite electric field formed from the polarization of molecules and molecular ions. Rad. Phys. Chem., $85,36-41$.

Moribayashi, K. (2013b). Demonstrations for the effect of composite electric fields of molecular ions on the motion of secondary electrons due to ion irradiation. Nucl. Instru. Meth. Phys. Res. B, 314C, 30-33. 
Moribayashi, K. (2014a). Radial dose calculation due to the irradiation of a heavy ion: Role of composite electric field formed from the polarization of molecules and molecular ions. Rad. Phys. Chem., 96, 211-216.

Moribayashi, K. (2014b). Relaxation of Plasma Created from Irradiation of a Heavy Ion. JPS Conference Proceedings, 1, 013089/1-4.

Moribayashi, K. (2015a). Development of the radial dose distribution function relevant to the treatment planning system for heavy particle cancer therapy. Phys. Scr., 90, 054013/1-5.

Moribayashi, K. (2015b). Simulation study of radial dose due to the irradiation of a swift heavy ion aiming to advance the treatment planning system for heavy particle cancer therapy: the effect of emission angles of secondary electrons. Nucl. Instru. Methods Phys. Res. B, 365, 592-595.

Nikjoo, H., Uehara, S., Wilson, W.E., Hoshi, \& M., Goodhead, D.T., (1998). Track structure in radiation biology: theory and applications. Int. J. Radiat. Biol., 73, 355-364.

Ohsawa, D., Tawara, H., Soga, F., Galassi, M.E., \& Rivarola, R.D., (2013). $6.0 \mathrm{MeVu}^{-1}$ carbon ion $\left(\mathrm{C}^{6+}\right.$ and $\mathrm{C}^{4+}$ )-induced secondary electron emission from water vapor. Phys. Scr., T156, 014039/1-5.

Orient, O.J. \& Srivastava, S.K., (1987). Electron impact ionisation of $\mathrm{H}_{2} \mathrm{O}, \mathrm{CO}, \mathrm{CO}_{2}$ and $\mathrm{CH}_{4}$. J. Phys. B., 20, 3923-3936.

Pritchard, H. P., McKoy, V., \& Lima, M. A. P. (1990). Electronic excitation of $\mathrm{H}_{2} \mathrm{O}$ by low-energy electron impact. Phys. Rev. A., 41, 546-549.

Rawat, P., Prabhudesai, V. S., Aravind, G., Rahman, M. A., \& Krishnakumar, E., (2007). Absolute cross sections for dissociative electron attachment to $\mathrm{H}_{2} \mathrm{O}$ and $\mathrm{D}_{2} \mathrm{O}$. J. Phys.B., 40, 4625-4636.

Rodberg, R. L. S. \& Thaler, R. M. (1967). Introduction to the Quantum Theory of Scattering. New York and London: Academic Press INC.

Rudd, M. E., Kim, Y. K., Madison, D. H., \& Gay, T. J. (1992). Electron production in proton collisions with atoms and molecules: energy distributions. Rev. Mod. Phys., 64, 441-490.

Uehara, S., \& Nikjoo, H., (2002). Monte Carlo Track Structure Code for Low-Energy Alpha-Particles in Water. J.Phys.Chem. B., 106, 11051-11063.

Varma, M. N., Baum, J. W., \& Kuehner, A. V. (1997). Radial dose, LET, and W for ${ }^{16} \mathrm{O}$ ions in $\mathrm{N}_{2}$ and Tissue equivalent gasses. Radiation Res., 70, 511-518.

Wang, H., \& Vassiliev, O. N. (2014). Radial dose distributions from protons of therapeutic energies calculated with Geant4-DNA. Phys. Med. Biol., 59, 3657-3668.

Warman, J. M., Sennhauser, E. S., \& Armstrong, D. A. (1979). Three body electron-ion recombination in molecular gases J. Chem. Phys., 70, 995-999.

Ziegler, J. F., Biersack, J. P., \& Ziegler, M. D. (2008). SRIM: The Stopping and Range of Ions in Matter. SRIM Co. Chap. (pp. 1-3).

\section{Copyrights}

Copyright for this article is retained by the author(s), with first publication rights granted to the journal.

This is an open-access article distributed under the terms and conditions of the Creative Commons Attribution license (http://creativecommons.org/licenses/by/3.0/). 